\title{
Retrieval of Total Scattering Cross Sections of Molecules from Inhomogeneously Broadened Absorption Lines
}

\author{
V. P. Kochanov $\mathbb{D}^{1}$ and L. N. Sinitsa $\mathbf{a}^{2,3}$ \\ ${ }^{1}$ Laboratory of Theoretical Spectroscopy, V.E. Zuev Institute of Atmospheric Optics, Siberian Branch, \\ Russian Academy of Sciences, 1, Academician Zuev Square, 634021 Tomsk, Russia \\ ${ }^{2}$ Laboratory of Molecular Spectroscopy, V.E. Zuev Institute of Atmospheric Optics, Siberian Branch, Russian Academy of Sciences, \\ 1, Academician Zuev Square, 634021 Tomsk, Russia \\ ${ }^{3}$ Department of Physics, Tomsk State University, 36, Lenin Ave., 634050 Tomsk, Russia
}

Correspondence should be addressed to V. P. Kochanov; koch@iao.ru

Received 20 June 2018; Revised 18 July 2018; Accepted 28 July 2018; Published 15 October 2018

Academic Editor: Jose S. Camara

Copyright (c) 2018 V. P. Kochanov and L. N. Sinitsa. This is an open access article distributed under the Creative Commons Attribution License, which permits unrestricted use, distribution, and reproduction in any medium, provided the original work is properly cited.

\begin{abstract}
A new method of retrieving quantitative information on the hard, soft, and diffraction collision's frequencies from inhomogeneously pressure-broadened line profiles was proposed and tested. The essence of the method lies in the processing of recorded profiles using various profiles' models containing these frequencies treated as adjustable parameters in different manners. Three self-broadened $\mathrm{H}_{2} \mathrm{O}$ absorption lines free of an instrumental function were processed with the use of four model line profiles. Retrieved set of pressure line broadening and narrowing constants allows finding out the sought frequencies. The estimation of the total scattering cross section, $\sim 630 \AA^{2}$, in pure water vapor was made.
\end{abstract}

\section{Introduction}

Various models for spectral line profiles allow retrieving information on collision processes that affect the line shape. The simplest Lorentzian and Voigt profiles provide retrieving only the homogeneous collision line half-width $\gamma$ and the line shift $\Delta$. With the aid of more complicated profiles [1-4], where the Dicke line narrowing $[5,6]$ is taken into account, it is possible to find out an additional parameter-the frequency of elastic velocity-changing collisions. In the conventional approach [1-6], this frequency is expressed via the diffusion coefficient $D$ as

$$
v_{h}=\frac{\bar{v}^{2}}{(2 D)},
$$

where $\bar{v}$ is the most probable thermal speed of absorbing molecules. Thus, exploitation of the collision line narrowing makes it possible to obtain the data on $D$ from processing recorded line profiles, which is still nonconventional information for spectroscopy. Similarly, involvement of other physical mechanisms of forming the line shape increases the number of the profile's adjustable parameters. Certain parameters may have a direct physical meaning, and thus they can serve as a source of valuable additional information extracted from a line shape. Such are the frequencies of soft and hard velocity-changing collisions.

Let us detail the latter assertion. The matter is that the structure of the collision integral implies division of its kernel onto three parts related to hard (hard collisions are mainly large-angle scattering collisions [9] that lead to establishing the equilibrium distribution of molecules over velocities after each collision, soft collisions are caused by classical scattering on small angles less $0.3 \mathrm{rad}$ [10], and the diffraction-scattering collisions occur when scattering angles are $\sim 0.01 \mathrm{rad}$ [8-10].), soft, and diffraction-scattering collisions [7-11]. These three types of collisions function to a great extent additively due to considerable difference in widths of the corresponding differential scattering cross sections. Consequently, the total output frequency of the collision integral $v_{t}$ can be represented as a sum [7-11]. 


$$
v_{\mathrm{t}}=\gamma+v_{\mathrm{h}}+v_{\mathrm{s}}+v_{\mathrm{d}}
$$

where $v_{\mathrm{h}}, v_{\mathrm{s}}$, and $v_{\mathrm{d}}$ are the input collision integral frequencies of the elastic hard, soft, and diffraction scattering collisions, respectively, and the Lorentzian collision halfwidth at half-height $\gamma$ contains the contributions of elastic and inelastic collisions. If the above frequencies enter into a model collision integral explicitly, then the line profile derived from the respective muster equation for a density matrix will contain these frequencies as adjustable parameters $[9,10]$. This opens a possibility to retrieve the newly introduced parameters from processing experimental line profiles.

It was shown earlier [12-14] that the output frequency $v_{\mathrm{t}}$ can be measured directly as a collision-broadening constant of nonlinear resonances at sufficiently low gas pressures. The theory $[14,15]$ allows expressing the frequency $v_{t}$ through the total collision cross section. Thus, qualitatively new fundamental information can be obtained from nonlinear spectroscopic measurements. On the contrary, in this paper, we intend to show that the value of $v_{t}$ also may be obtained by means of linear spectroscopy. Namely, correct account of the combined effect of hard and soft collisions [8-11] performed on the basis of the quantum-mechanical collision integral kernel [7] and the inverse-power intermolecular interaction potentials provides the presence of all the frequencies specified in (2) in the model line profiles [8-11]. Therefore, in principle, it is possible to retrieve these frequencies from processing recorded spectra with the profiles [8-11]. Though, such retrieval is not guaranteed to be a priori successful because of a strong correlation between some of the profile's parameters [10]. At the same time, certain parameters of the collision integral can be calculated theoretically for different intermolecular interaction potentials [9, 10]. In particular, such is the ratio of soft to hard collision frequencies, which also can be used in the fitting. Thus, we can assume that the ambiguity caused by marked above correlation of the parameters can be eliminated, and the retrieving different collision integral frequencies will be possible. Ascertainment of a principal scheme of the estimation of the discussed frequencies and testing its operability form the goals of this paper.

\section{Experimental Setup}

The high-resolution spectra of $\mathrm{H}_{2} \mathrm{O}$ were recorded with the use of the photoacoustic (PhA) spectrometer based on a single-mode Ti-sapphire laser pumped by an Ar-ion laser. The Ti-sapphire laser had the output power of $1 \mathrm{~W}$ and the $50 \mathrm{kHz}$ linewidth. It was able to tune in the 11300 $12800 \mathrm{~cm}^{-1}$ spectral range with the $20 \mathrm{MHz}$ frequency step. This provided the spectral resolution better than $0.001 \mathrm{~cm}^{-1}$ and the negligible contribution of the laser bandwidth to recorded line profiles (the Doppler HWHM was $\sim 0.018 \mathrm{~cm}^{-1}$ or $542 \mathrm{MHz}$ ). The high power of the laser provided a high threshold absorption sensitivity of the spectrometer, $\sim 1 \cdot 10^{-8} \mathrm{~cm}^{-1}$. The body of the PhA cell was made of stainless steel. The length of the cell was $10 \mathrm{~cm}$. The acoustic signal in the PhA cell was detected by high-sensitive laboratory-made capacitor microphone with a preamplifier. A signal of the photoacoustic detector is directly proportional to the absorption coefficient of the gas as opposed to the spectrophotometric method where a signal is proportional to the transmittance of the medium. This circumstance allows recording a weak absorption with a higher accuracy. Wavelength calibration was carried out with the use of the Fourier spectrometer "Bruker DA 003". The measurement technique is described in more detail in $[16,17]$.

Three $\mathrm{H}_{2} \mathrm{O}$ lines centered at 12415.204, 12413.977, and $12411.404 \mathrm{~cm}^{-1}(\approx 805 \mathrm{~nm})$ were used to perform the estimations outlined in Introduction. Parameters of these lines taken from [18] are presented in Table 1. The self-broadening of these lines was registered within the pressure range 1 Torr $\leq p \leq 20$ Torr.

\section{Data Processing}

The following line profiles have been used in data processing:

(i) $K_{\mathrm{HC}}(\Omega)$-the conventional profile in the hard collision model $[3,4]$ (calculated with equations (3) and (8) in [19]; $\Omega$ is the frequency detuning from a line center).

(ii) $K_{\mathrm{RS} 3}(\Omega)$-the profile based on the quantummechanical Rautian-Shalagin collision integral kernel [7] including the speed dependence [20,21] and calculated for the dipole-dipole intermolecular interaction potential $\alpha r^{-3}$, where $r$ is the distance between colliding molecules (equations (A1) and (A2) in [9]).

(iii) $K_{\mathrm{SH} 3}(\Omega)$ - the profile accounting for the speeddependence, soft, and hard velocity-changing collisions and based on a collision integral partly represented by a differential form with the use of some fixed parameters calculated for dipole-dipole intermolecular interaction (equation (9) and Table 1 in [10]; equations (5) and (6) in Ref. [22]).

(iv) $K_{S H 3 r}(\Omega)$-the same profile as the previous one where the ratio of soft to hard collision frequencies $r$ is set as an adjustable parameter (equation (9) and Table 1 in [10]; equations (5) and (6) in [22]).

This group of profiles may provide retrieving all the collision frequencies presented in (2). Really, the half-width $\gamma$ is obtained with each of the profiles listed above. If $\gamma$ is defined as a quantity averaged over velocities of absorbing molecules, then, as follows from the practice [23], its values obtained with the use of all the profiles will be equal with a satisfactory accuracy. The dimensionless collision linenarrowing parameter is as follows:

$$
\alpha=\frac{v_{h}}{\gamma}
$$

which is the fitting parameter of the profiles (i), (iii), and (iv), while the profile (ii) contains the substantially different narrowing parameter 
TABLE 1: Line parameters taken from [18].

\begin{tabular}{lcccc}
\hline$v_{0}\left(\mathrm{~cm}^{-1}\right)$ & Band & $J^{\prime \prime} K_{a}^{\prime \prime} K_{c}^{\prime \prime}$ & $J^{\prime} K_{a}^{\prime} K_{c}^{\prime}$ & $\begin{array}{c}10^{25} S \\
(\mathrm{~cm} / \text { molecule })\end{array}$ \\
\hline 12415.20199 & $\nu_{2}+3 \nu_{3}$ & 524 & 625 & 6.48 \\
12413.94479 & $3 v_{1}+\nu_{2}$ & 634 & 541 & 1.4 \\
12411.40028 & $\nu_{2}+3 v_{3}$ & 514 & 615 & 2.53 \\
\hline
\end{tabular}

Single and double strokes denote lower and upper molecular states, respectively; $S$ is the line intensity.

$$
\alpha_{\text {tot }}=\frac{\left(\nu_{\mathrm{h}}+\nu_{\mathrm{s}}+\nu_{\mathrm{d}}\right)}{\gamma} \text {. }
$$

As is seen from the comparison of (3) and (4), the inequality $\alpha_{\text {tot }}>\alpha$ always holds under the natural supposition of $\nu_{\mathrm{h}}, v_{\mathrm{s}}$, and $\nu_{\mathrm{d}}>0$. The relative difference $\alpha_{\mathrm{tot}} / \alpha-1$ may be significant, and it can amount hundreds of percent [11]. Note that $\alpha$ describes an actual line narrowing caused by collisions with a molecular scattering on predominantly large angles [9]. Due to this reason, the values of $\alpha$ obtained with the aid of the profiles (i), (iii), and (iv) must be approximately equal. In contrast to this, the parameter $\alpha_{\text {tot }}$ was introduced in [8] as the ratio of the sum frequency of elastic velocity-changing collisions, $\nu_{\mathrm{h}}+v_{\mathrm{s}}+\nu_{\mathrm{d}}$, to a homogeneous line half-width $\gamma$. Accepted in $[8,9]$, parametrization allows interpretation of $\alpha_{\text {tot }}$ as a fitting parameter and further retrieving the values of this narrowing parameter from spectra processing with profiles $[8,9]$.

The ratio

$$
r=\frac{v_{\mathrm{s}}}{v_{\mathrm{h}}}
$$

can be calculated theoretically $[9,10]$ and then used as a fixed parameter in profile (iii). Otherwise, $r$ is a fitting parameter of profile (iv).

We note that the contour (i) does not contain parameters that are complementary to the parameters of the remaining three contours, and its application in processing is justified by controlling the values of $\gamma$ and $\alpha$ obtained with the help of the profiles (ii)-(iv) (see Table 2).

The knowledge of values of $\gamma, \alpha, \alpha_{\text {tot }}$, and $r$ retrieved with the aid of fitting the profiles (i)-(iv) to experimental ones makes it possible to solve the system of Equations (2)-(5) with respect to the sought frequencies $v_{\mathrm{t}}, v_{\mathrm{h}}, v_{\mathrm{s}}$, and $v_{\mathrm{d}}$. This solution gives a fundamental scheme of quantitative estimation of these frequencies. The respective expressions for the frequencies are

$$
\begin{aligned}
v_{t} & =\left(1+\alpha_{t o t}\right) \gamma, \\
v_{h} & =\alpha \gamma, \\
\nu_{s} & =r \alpha \gamma, \\
\nu_{d} & =\left[\alpha_{t o t}-(1+r) \alpha\right] \gamma .
\end{aligned}
$$

An additional testing these frequencies can be done by means of comparison of the ratios

$$
R=\frac{v_{s}+v_{d}}{v_{h}+v_{s}+v_{d}}
$$

found from the experiment and relations (6) with the calculated values of $R$, presented in Figure 2 of [9].

It should be noted that the values of the collision line half-width $\gamma$ retrieved from an experiment with the use of profiles (i)-(iv) distinguish due to different manners of accounting the speed dependence in them. But in cases when the speed dependence is small pronounced (heavy absorbing and/or light perturbing molecules), discrepancies between $\gamma$ are minor [23], Table 2. Therefore, the main source of errors in the suggested method of retrieving frequencies $v_{\mathrm{t}}, v_{\mathrm{h}}, v_{\mathrm{s}}$, and $\nu_{\mathrm{d}}$ lies in an inaccuracy of the narrowing parameters $\alpha$ and $\alpha_{\text {tot }}$, as well as the ratio of the frequencies of soft and hard collisions $r$, which are more sensitive characteristics of a line shape.

An example of a recorded line with the best-fitted profile $K_{\mathrm{HC}}(\Omega)$ is shown in Figure 1. The quality of fitting all the rest of listed above profiles is practically the same because of the account of the line narrowing in each of them, which makes these profiles to be flexible enough. The collision line broadening found from the processing is presented in Figure 2. Straight lines in this figure are plotted as a result of least-squares fitting and their, slopes represent the pressurebroadening coefficients $\gamma_{0} \equiv \gamma / p$. A complete set of the parameters retrieved from the processing is presented (hard collisions are mainly large-angle scattering collisions [9] that lead to establishing the equilibrium distribution of molecules over velocities after each collision; soft collisions are caused by classical scattering on small angles less $0.3 \mathrm{rad}$ [10], and the diffraction scattering collisions occur when scattering angles are $\sim 0.01 \mathrm{rad}$ [8-10].) (Except for line intensities $S$ disclosing wide range of values, which is typical for PhA measurements.) in Table 2. From this table and Equations (6) and (7), we have obtained the estimated values of the collision integral frequencies (Table 3 ).

\section{Discussion and Conclusions}

It is seen from Table 2 that the pressure-broadening coefficients $\gamma_{0}$ for individual lines obtained with different line profiles are in a good agreement. Also it should be noted that the narrowing parameters $\alpha$ (2) are small compared with the values found with the aid of the expression for $\nu_{h}(1)$ via the self-diffusion coefficient $D$. In particular, the use of the literature data $D=0.196 \mathrm{~cm}^{2} / \mathrm{s}$ [24] and $\gamma_{0}=18 \mathrm{MHz} /$ Torr taken from Table 2 leads to $\alpha=0.51$ that is two and a half times greater than the values of $\alpha \sim 0.2$ retrieved from the experiment. This fact can be understood on the basis of simple physical arguments. Indeed, for long-range intermolecular interactions (i.e., dipole-dipole and dipolequadrupole ones), the contribution of the molecular scattering on small angles in collision integral frequencies increases relatively to the scattering on large angles (see Figure 2 in Ref. [9]). In other words, the large-angle scattering is suppressed. Correspondingly, as is seen from Table 2, the values of the parameter $\alpha_{\text {tot }}$ (4) including frequencies of scattering on small angles are large compared with the values of $\alpha$ (3). Due to the decrease in the hard velocity-changing collisions, the Dicke line narrowing 
TABLE 2: Line parameters retrieved from data processing with the use of various model line profiles. The numerals in parenthesis correspond to last significant digits of the left numbers and they are 95-percent confidential intervals.

\begin{tabular}{|c|c|c|c|c|c|c|}
\hline$\nu_{0}[18]\left(\mathrm{cm}^{-1}\right)$ & Model & $\gamma_{0}(\mathrm{MHz} /$ Torr $)$ & $\alpha$ & $\alpha_{\text {tot }}$ & $\Delta_{0}(\mathrm{MHz} /$ Torr $)$ & $r$ \\
\hline \multirow{5}{*}{12415.20199} & $\mathrm{HC}$ & $18.00(16)$ & $0.12(5)$ & - & $-5.9(1.1)$ & - \\
\hline & RS3 & $18.01(15)$ & $0.12(5)$ & $9.0(3.2)$ & $-5.9(1.1)$ & - \\
\hline & $\mathrm{SH} 3$ & $19.37(36)$ & $0.22(8)$ & - & $-5.9(1.1)$ & 2.1 \\
\hline & $\mathrm{SH} 3 \mathrm{r}$ & $18.03(17)$ & $0.20(8)$ & - & $-5.9(1.1)$ & $1.4(5)$ \\
\hline & & $18.89[18]$ & - & - & - & - \\
\hline \multirow{5}{*}{12413.94479} & $\mathrm{HC}$ & $11.83(99)$ & $0.0(2)$ & - & $6.3(3)$ & - \\
\hline & RS3 & $10.59(38)$ & $0.12(3)$ & $8.5(1.7)$ & $6.3(3)$ & - \\
\hline & SH3 & $12.2(1.3)$ & 0.19 (15) & - & $6.3(3)$ & 2.1 \\
\hline & $\mathrm{SH} 3 \mathrm{r}$ & $11.7(2.1)$ & $0.12(19)$ & - & $6.3(3)$ & $1.7(8)$ \\
\hline & & $16.69[18]$ & - & - & - & - \\
\hline \multirow{5}{*}{12411.40028} & $\mathrm{HC}$ & $18.72(12)$ & $0.07(2)$ & - & $7.9(9)$ & - \\
\hline & RS3 & $18.72(12)$ & $0.07(2)$ & $5.3(1.2)$ & $7.9(9)$ & - \\
\hline & SH3 & $18.99(1)$ & $0.13(4)$ & - & $7.9(9)$ & 2.1 \\
\hline & $\mathrm{SH} 3 \mathrm{r}$ & $19.23(18)$ & $0.38(23)$ & - & $7.9(9)$ & $2.4(1.2)$ \\
\hline & & $21.06[18]$ & - & - & - & - \\
\hline
\end{tabular}

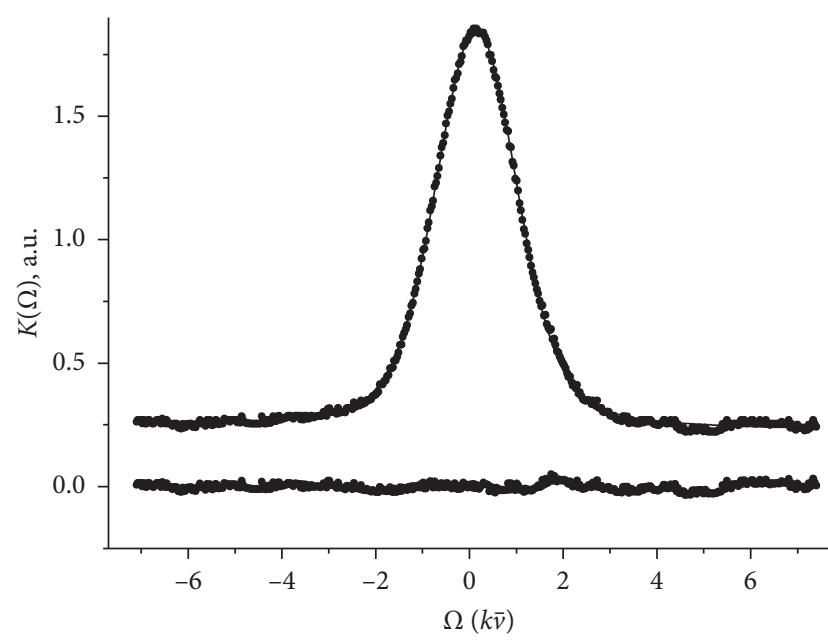

Figure 1: An example of recorded line profiles (points) and the best-fitted $K_{\mathrm{HC}}$ profiles (solid lines). Points below are the difference between the experiment and the theory. The corresponding differences between the experiment and profiles (ii)-(iv) are practically indistinguishable. $\nu_{0}=12413.94479 \mathrm{~cm}^{-1}, p_{\mathrm{H}_{2} \mathrm{O}}=14.94$ Torr.

controlled by the parameter $\alpha$ also reduces. Thus, it is evident that relation (1) cannot be used for a reliable quantitative retrieving $D$ from experimental values of narrowing parameters in the case of long-range intermolecular interactions.

Note that the parameters $r$ (5) in Table 2 retrieved with the line profile $K_{\mathrm{SH} 3 \mathrm{r}}$ have the same order of magnitude as the relating calculated values of $r$ [10] used in fitting with the profile $K_{\mathrm{SH} 3}$.

Discussing Table 3, we mark that the main contribution to $v_{\mathrm{t}}$ is made by $\nu_{\mathrm{d}}$. In accordance with the above discussion, this fact can be imputed to the long-range action of the dipole-dipole intermolecular interaction potential of the water molecules having a large constant dipole moment.

There is an interesting opportunity to estimate the total scattering cross section $\sigma$ from data on $v_{\mathrm{t}}$ presented in Table 3, namely, equations (15)-(18) in [15], where the collision integral frequencies are expressed through the parameters of the intermolecular potential $U_{\text {jn }}(r)=-C_{\text {jn }} / r^{n}$

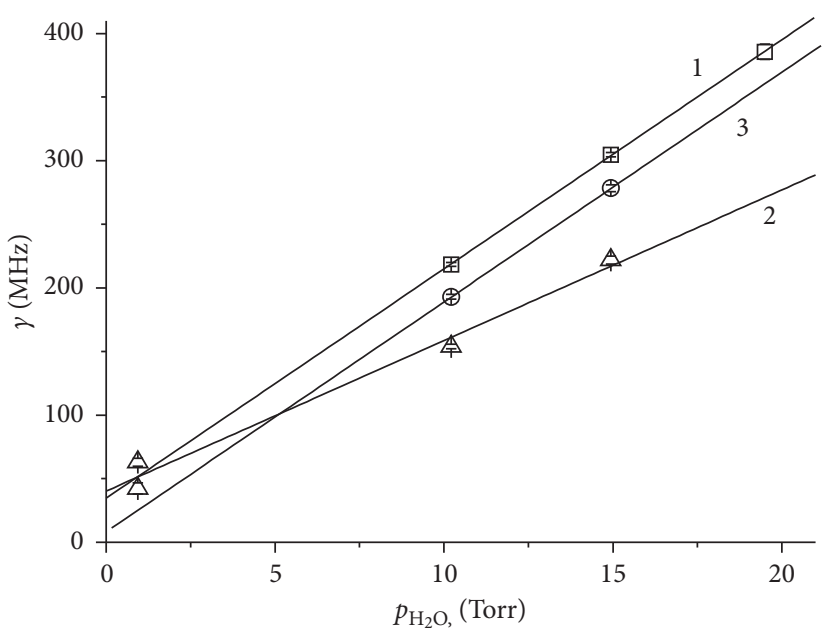

FIgURE 2: Dependence of collision HWHM $\gamma$ on pressure of $\mathrm{H}_{2} \mathrm{O}$. $\nu_{0}=12415.20199 \mathrm{~cm}^{-1}$ (straight line 1), $12413.94479 \mathrm{~cm}^{-1}$ (2), and $12411.40028 \mathrm{~cm}^{-1}$ (3). Symbols inside squares, circles, and triangles represent $95 \%$ confidence intervals.

(here $j$ denotes a molecular state), allow expressing the real part of $v_{\mathrm{t}}$ via $\sigma$ averaged over velocities. Let us assume that constants $C_{\text {jn }}$ are approximately equal for the upper and lower states of the considered vibrational-rotational transition [24]. Then, the index $j$ can be omitted, and we have the following expression from equations (15) and (16) of [15] and the optical theorem of scattering [25]:

$$
\begin{aligned}
\sigma & =\frac{4 \pi \hbar}{\mu u} \operatorname{Im}[f(\mathbf{u}, \mathbf{u})]=4 \int_{0}^{\infty} \rho \sin ^{2}\left(\frac{\rho_{w}}{\rho}\right)^{n-1} d \rho \\
& =2^{2 /(n-1)-1} \pi \frac{\csc (\pi /(n-1))}{\Gamma(2 /(n-1))}\left(\frac{\bar{v}}{u}\right)^{1 /(n-1)} \rho_{w}^{2}, \\
\rho_{w} & =2^{-1 /(n-1)} \pi^{1 / 2(n-1)}\left[\frac{C_{n} \Gamma((n-1) / 2)}{\hbar \bar{v} \Gamma(n / 2)}\right]^{1 /(n-1)} .
\end{aligned}
$$


TABLE 3: Collision integral frequencies in units of respective values of $\gamma$ retrieved from data processing and Equation (6).

\begin{tabular}{lccccc}
\hline$\nu_{0}\left(\mathrm{~cm}^{-1}\right)$ & $\nu_{\mathrm{d}} / \gamma$ & $\nu_{\mathrm{s}} / \gamma$ & $\nu_{\mathrm{h}} / \gamma$ & $\nu_{\mathrm{t}} / \gamma$ & $R$ \\
\hline 12415.19306 & $8.6 \pm 3.0$ & $0.23 \pm 0.14$ & $0.17 \pm 0.04$ & $10.0 \pm 3.2$ & $0.98 \pm 0.01$ \\
12413.96286 & $8.1 \pm 1.4$ & $0.24 \pm 0.18$ & $0.14 \pm 0.04$ & $9.5 \pm 1.7$ & $0.98 \pm 0.01$ \\
12411.39004 & $4.8 \pm 0.7$ & $0.4 \pm 0.4$ & $0.16 \pm 0.10$ & $6.3 \pm 1.2$ & $0.97 \pm 0.03$ \\
\hline
\end{tabular}

where $f(\mathbf{u}, \mathbf{u})$ is the amplitude of elastic scattering on zero angles, $\rho_{w}$ is the Weisskopf radius, $\mathbf{u}$ is the relative velocity of molecules in the center-of-mass coordinate system, and $\Gamma$ is the gamma function.

Averaging $\sigma(8)$ over $\mathbf{u}$ with the Maxwellian distribution of relative velocities gives

$$
\begin{aligned}
\sigma_{a v} & =2^{2 /(n-1)} \sqrt{\pi}\left(\frac{\beta}{\beta+1}\right)^{1 / 2(n-1)} \frac{\csc (\pi /(n-1)) \Gamma(3 / 2-1 /(2 n-2))}{\Gamma(2 /(n-1))} \rho_{w}^{2}, \\
\beta & \equiv \frac{m_{b}}{m},
\end{aligned}
$$

where $m$ and $m_{\mathrm{b}}$ are masses of absorbing and perturbing molecules, respectively.

Analogous averaging real part of the output collision integral frequency given by equation (18) in [15] over velocities of absorbing molecules leads to

$$
\nu_{\mathrm{av}}=2^{2-2 /(n-1)} \frac{\beta^{1 /(n-1)-1 / 2}}{\sqrt{\beta+1}} \frac{(n-2) \Gamma(1-1 /(n-1))}{(n-1)} \pi N_{\mathrm{b}} \bar{v} \rho_{w}^{2},
$$

where $N_{\mathrm{b}}$ is the density of perturbing molecules.

After combining (9) and (10), we have the expression of the average total scattering cross section through the output collision integral frequency:

$$
\sigma_{\text {tot }}=\frac{2^{5 /(n-1)-3} \csc (\pi /(n-1))}{\Gamma(1-1 /(2 n-2)) \Gamma(2 /(n-1))}[\beta(\beta+1)]^{(n-2) / 2(n-1)} \frac{\nu_{\mathrm{av}}}{N_{\mathrm{b}} \bar{v}} \text {. }
$$

In particular, in the considered case of self-broadening $\mathrm{H}_{2} \mathrm{O}$ lines (dipole-dipole interaction with $n=3$ and $\beta=1$ ), it follows from (11):

$$
v_{a v}=1.457 N_{b} \bar{v} \sigma_{t o t} .
$$

Calculations performed on the basis of (12) and the data of Tables 2 and 3 give the following estimations for the total scattering cross section for $\mathrm{H}_{2} \mathrm{O}-\mathrm{H}_{2} \mathrm{O}$ collisions: $\sigma_{\text {tot }} \sim$ $630 \pm 150 \AA^{2}$. For comparison, the typical values of gaskinetic cross sections of many molecules are of the order 50-70 $\AA^{2}$ [26]. Relatively large errors (95\% confidence intervals) in $\sigma_{\text {tot }}$ may be caused by insufficiently accurate experiment as well as by a rough net used in numerical integration over velocities in the vicinity of a character point determining diffraction scattering [9]. The above estimation of $\sigma_{\text {tot }}$ for $\mathrm{H}_{2} \mathrm{O}$ molecule is close to total cross sections of three other molecules obtained by direct measurements of $\nu_{\mathrm{t}}$ with the aid of nonlinear spectroscopy methods [12, 13, 27-30], namely, analogous calculations made in [27] ascertain that in pure gas of $\mathrm{CH}_{4}, \sigma_{\text {tot }} \approx 380$
[28], 350 [12], and $370 \AA^{2}$ [29]; in $\mathrm{CO}_{2} \sigma_{\text {tot }} \approx 500 \AA^{2}[13]$, and in $\mathrm{SF}_{6} \sigma_{\text {tot }} \approx 600 \AA^{2}[30]$.

Coming to the conclusions, it can be stated that the proposed new method of retrieving input and output collision integral frequencies is mainly functional. It was shown that with the aid of this method, it is possible to get estimations of a fundamental quantity-total scattering cross section. At the same time, the results presented in this paper are only first steps in the outlined direction, and retrieving more accurate quantitative data on the frequencies and cross sections requires further improvement of experiment and theory, which can constitute a significant cycle of work in the spectroscopy of the line contour.

From the data processing, it was found that relation (1) between the frequency of hard collisions and the diffusion coefficient fails in the case of long-ranged intermolecular interactions.

\section{Data Availability}

The data used to support the findings of this study are included within the article.

\section{Conflicts of Interest}

The authors declare that they have no conflicts of interest.

\section{Acknowledgments}

One of the authors (L. Sinitsa) expresses his gratitude to coworkers from the University of Science and Technology (Hefei, China) for the possibility to use Ti-sapphire laser. Dr. S. N. Mikhailenko is acknowledged for the help in compiling Table 1.

This work was partly supported by the RFBR (Grant No. 16-43-700492).

\section{References}

[1] L. Galatry, "Simultaneous effect of Doppler and foreign gas broadening on spectral lines," Physical Review, vol. 122, no. 4, pp. 1218-1223, 1961.

[2] M. I. Podgoretsky and A. V. Stepanov, "On the Doppler emission and absorption line width," Jurnal eksperimental'noi i teoreticheskoi fiziki, vol. 40, no. 2, pp. 561-566, 1961.

[3] M. Nelkin and A. Ghatak, "Simple binary collision model for Van Hove's Gs(r,t)," Physical Review, vol. 135, no. 1A, pp. A4-A9, 1964.

[4] S. G. Rautian and I. I. Sobel'man, "The effect of collisions on the Doppler broadening of spectral lines," Soviet Physics Uspekhi, vol. 9, no. 5, pp. 701-716, 1967. 
[5] R. H. Dicke, "The effect of collisions upon the Doppler width of spectral lines," Physical Review, vol. 89, no. 2, pp. 472-473, 1953.

[6] J. P. Wittke and R. H. Dicke, "Redetermination of the hyperfine splitting in the ground state of atomic hydrogen," Physical Review, vol. 103, no. 3, pp. 620-631, 1956.

[7] S. G. Rautian, G. I. Smirnov, and A. M. Shalagin, Nonlinear Resonances in Spectra of Atoms and Molecules, Nauka, Novosibirsk, SB, Russia, 1979.

[8] V. P. Kochanov, "Manifestations of small-angle molecular scattering in spectral line profiles," Journal of Experimental and Theoretical Physics, vol. 118, no. 3, pp. 335-350, 2014.

[9] V. P. Kochanov, "Combined effect of small- and large-angle scattering collisions on a spectral line shape," Journal of Quantitative Spectroscopy and Radiative Transfer, vol. 159, pp. 32-38, 2015.

[10] V. P. Kochanov, "Speed-dependent spectral line profile including line narrowing and mixing," Journal of Quantitative Spectroscopy and Radiative Transfer, vol. 177, pp. 261-268, 2016.

[11] V. P. Kochanov, "Effect of diffraction of molecules on collisional line narrowing," Optics and Spectroscopy, vol. 89, no. 5, pp. 684-689, 2000.

[12] S. N. Bagayev, E. V. Baklanov, and V. P. Chebotayev, "Measurement of elastic scattering cross-sections in a gas by laser spectroscopy methods," ZhETF Pis. Red., vol. 16, no. 1, pp. 15-18, 1972.

[13] V. P. Chebotayev, V. P. Kochanov, and L. S. Vasilenko, "Nonlinear dependence of optical resonance widths at $\mathrm{CO}_{2}$ transitions on pressure," Optics Communications, vol. 20, no. 3, pp. 409-411, 1977.

[14] V. P. Kochanov, S. G. Rautian, and A. M. Shalagin, "Broadening of nonlinear resonances by velocity-changing collisions," Journal of Experimental and Theoretical Physics, vol. 45, no. 4, pp. 714-722, 1977.

[15] V. P. Kochanov, "Line profiles for the description of line mixing, narrowing, and dependence of relaxation constants on speed," Journal of Quantitative Spectroscopy and Radiative Transfer, vol. 112, no. 12, pp. 1931-1941, 2011.

[16] V. Lazarev, Y. Ponomarev, L. Sinitsa, J.-X. Han, L.-Y. Hao, and Q.-S. Zhu, "The Photo-acoustic spectrometer with Ti: sapphire laser," SPIE Proceedings, vol. 3090, pp. 245-248, 1997.

[17] V. I. Serdyukov, L. N. Sinitsa, A. S. Dudaryonok, N. N. Lavrentieva, and A. P. Shcherbakov, "Investigation of the $\mathrm{H}_{2} \mathrm{O}$ line broadening using an optoacoustic laser spectrometer in the range of 12411-12421 $\mathrm{cm}^{-1}$," Russian Physical Journal, vol. 58, pp. 1-5, 2016.

[18] L. S. Rothman, I. E. Gordon, Y. Babikov et al., "The HITRAN2012 molecular spectroscopic database," Journal of Quantitative Spectroscopy and Radiative Transfer, vol. 130, pp. 4-50, 2013.

[19] V. P. Kochanov, "Algebraic approximation of the spectral line profile with allowance for hard and soft speed-related collisions," Atmospheric and Oceanic Optics, vol. 28, no. 5, pp. 394-399, 2015.

[20] P. R. Berman, "Speed-dependent collisional width and shift parameters in spectral line profiles," Journal of Quantitative Spectroscopy and Radiative Transfer, vol. 12, no. 9, pp. 13314132, 1972.

[21] J. Ward, J. Cooper, and E. W. Smith, "Correlation effects in the theory of combined Doppler and pressure broadening.-I. Classical theory," Journal of Quantitative
Spectroscopy and Radiative Transfer, vol. 14, no. 7, pp. 555-590, 1974.

[22] V. P. Kochanov, "On parameterization of spectral line profiles including the speed-dependence in the case of gas mixtures," Journal of Quantitative Spectroscopy and Radiative Transfer, vol. 189, pp. 8918-8923, 2017.

[23] V. P. Kochanov and I. Morino, "Methane line shapes and spectral line parameters in the 5647-6164 cm ${ }^{-1}$ region," Journal of Quantitative Spectroscopy and Radiative Transfer, vol. 206, pp. 313-322, 2018.

[24] V. P. Kochanov and V. I. Starikov, "Calculation and algebraic approximation of the diffusion and viscosity coefficients for polar gases as functions of the temperature and the parameters of the Stockmayer potential," Optika Atmosfery $i$ Okeana, vol. 14, no. 9, pp. 837-840, 2001.

[25] J. R. Taylor, Scattering Theory. The Quantum Theory of Nonrelativistic Collisions, John Wiley \& Sons, NY, London, Sydney, and Toronto, 1972.

[26] J. O. Hirschfelder, C. F. Curtiss, and R. B. Bird, Molecular Theory of Gases and Liquids, Wiley, New York, NY, USA, 1954.

[27] V. P. Kochanov and V. P. Lopasov, "Investigation of an absorption line profile in molecular gases with methods of laser spectroscopy," in Spectral Manifestations of Intermolecular Interactions in Gases, Y. S. Makushkin, Ed., Nauka, Novosibirsk, SB, Russia, pp. 142-172, 1982, in Russian.

[28] R. L. Barger and J. L. Hall, "Pressure shift and broadening of methane line at $3.39 \mu$, studied by laser-saturated molecular absorption," Physical Review Letters, vol. 22, no. 1, pp. 4-8, 1969.

[29] W. Radloff and V. Stert, "Contrast of an inverted Lamb dip of a laser with an internal absorption cell," Soviet Journal of Quantum Electronics, vol. 6, no. 7, pp. 817-821, 1976.

[30] N. G. Basov, O. N. Kompanets, V. S. Letokhov, and V. V. Nikitin, "Investigation of narrow resonances within the Doppler line of the rotational-vibrational transitions of the $\mathrm{SF}_{6}$ molecule during absorption saturation," Soviet Physics Journal of Experimental and Theoretical Physics, vol. 32, pp. 214-219, 1971. 

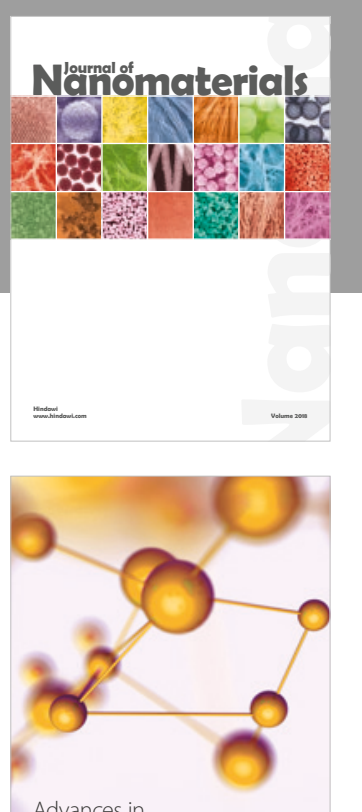

Physical Chemistry
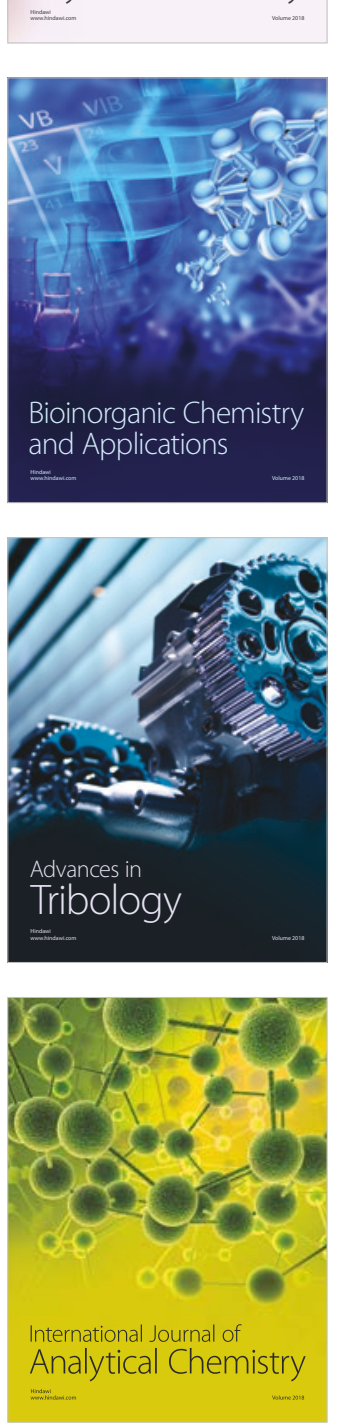

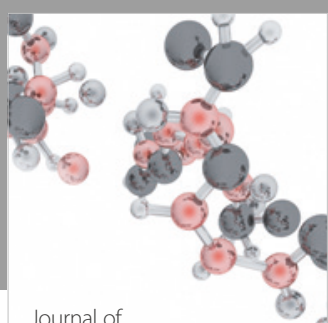

Analytical Methods

in Chemistry

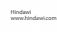

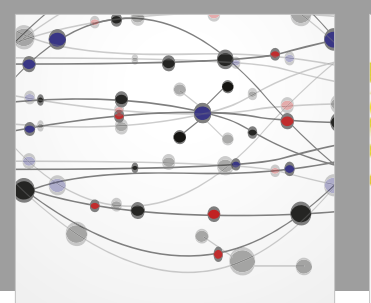

The Scientific World Journal

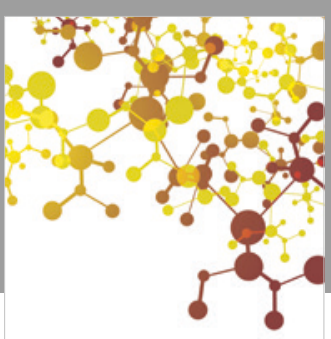

Journal of

Applied Chemistry
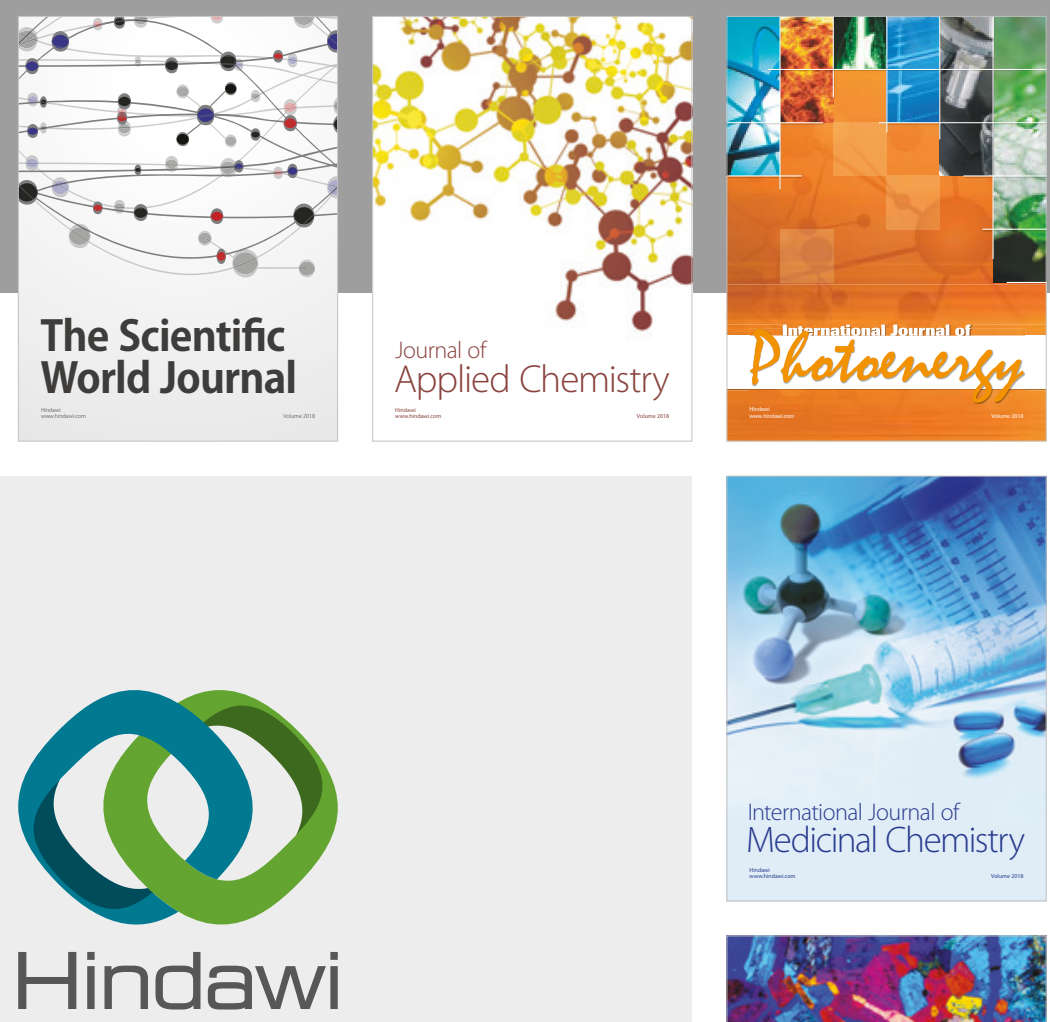

Submit your manuscripts at

www.hindawi.com
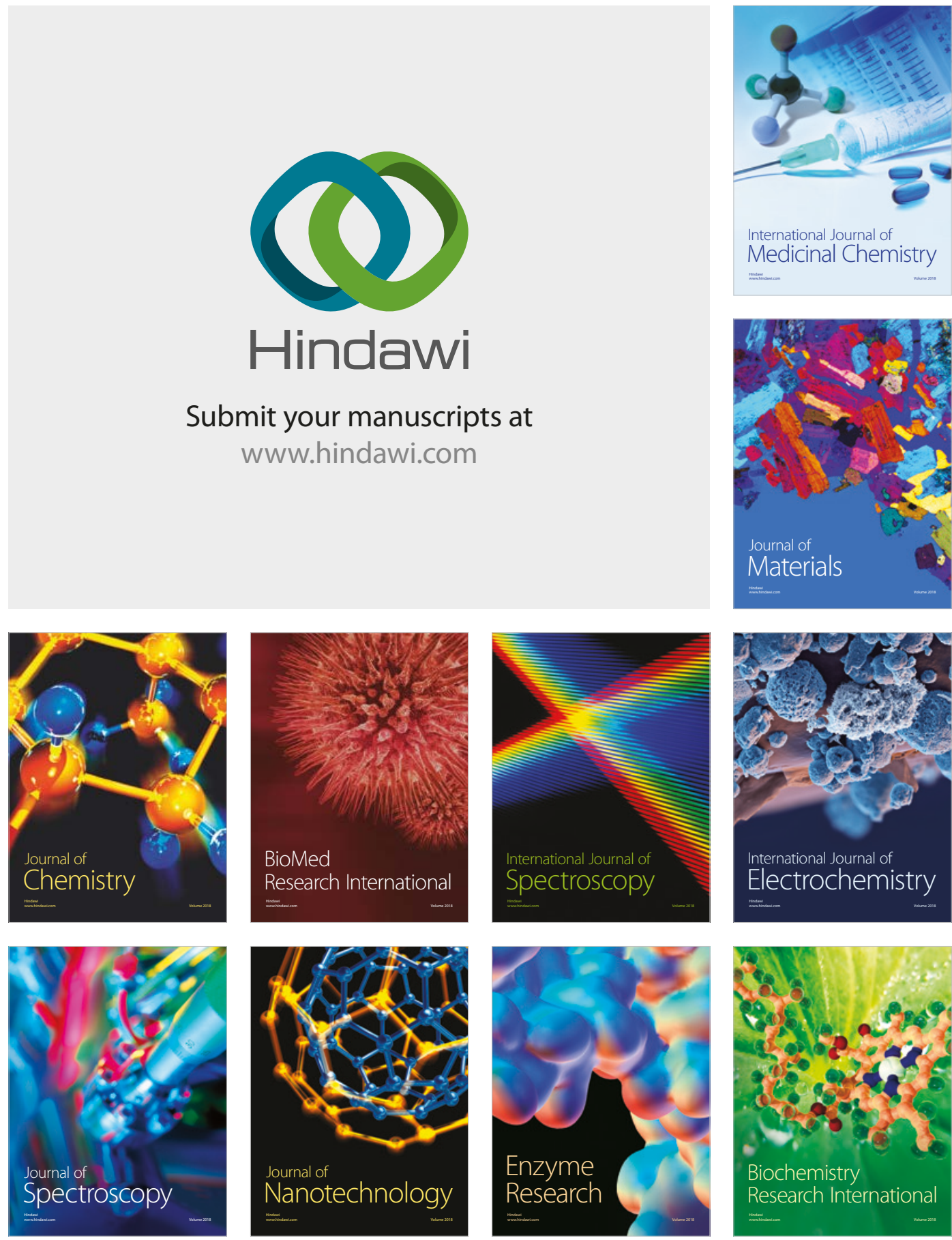
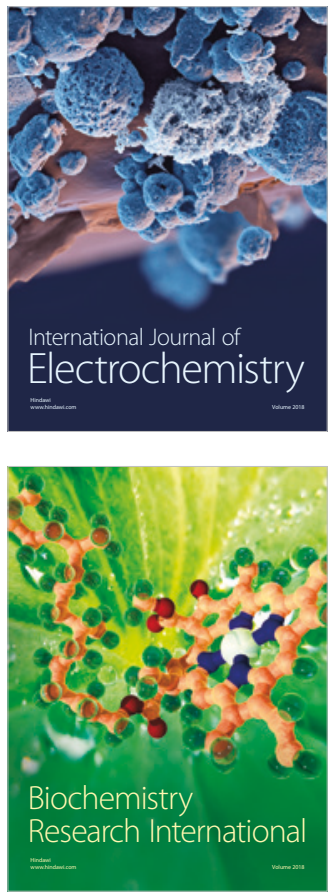